\title{
Evaluation of an extended period of use for preserved eye drops in hospital practice
}

\author{
D J Livingstone, G W Hanlon, S Dyke
}

\begin{abstract}
Aim-To evaluate and compare the microbial contamination arising from 1 and 2 weeks' use of eye drops by hospital inpatients and hence determine the validity of apportioning a 2 week in use expiry date for these preparations.

Methods-Eye drop residues were collected from inpatients of Worthing, Southlands, and Brighton General hospitals after 7 days' use (341 samples) and also after 14 days' use (295 samples). The contents of the containers were examined for the presence of contaminating bacteria and fungi.

Results-The incidence of microbial contamination was shown to be not significantly different $\left(p>0.1 \chi^{2}\right.$ test) between the 7 and 14 day samples. In addition, the contaminating micro-organisms were of a broadly similar pattern between the two sample groups and were mostly those normally associated with the skin. Less frequent contaminants were organisms of environmental origin. None of the microorganisms isolated were considered to be of clinical significance and the mean number of cells found per sample was very low.

Conclusions-The evidence therefore suggests that increasing the period of use for eye drops in hospitals from 7 to 14 days would not present a clinically significant threat to patients' health and yet may lead to annual savings to the NHS of $£ 0.5$ million.

(Br F Ophthalmol 1998;82:473-475)
\end{abstract}

Before 1966 there were no nationwide regulations governing the production, presentation, and duration of use of ophthalmic drug preparations. As a consequence of a number of serious incidents arising from the microbial contamination of eye drops, standards of pharmaceutical practice were published in the British Pharmaceutical Codex ${ }^{1}$ requiring eye drops to be:

“...sterile aqueous or oily suspensions ...prepared in a vehicle which is bactericidal and fungicidal. ...In general, a container of eye drops may be used for about one month for domiciliary purposes. When eye drops are used in hospital wards they should be discarded not later than one week after opening". These requirements were subsequently refined and adopted by the Department of Health and Social Security ${ }^{2}$ and remain in operation today.

While there is convincing evidence to support the sterile production of eye drops and the inclusion of an antimicrobial preservative, the pertinence of a 7 day expiry for hospital use appears to be rather less worthy. Extensive survey of the literature plus consultation with regulatory authorities has failed to reveal the scientific basis for the above directive. Indeed a preliminary study undertaken in Sweden in 1969 indicated that the sterility of eye drops supplied to hospital inpatients was maintained following up to 2 weeks' use and therefore a 2 week expiry might be deemed more appropriate. $^{3}$ It would therefore appear, that the assignment of in use expiry dates for eye drops are "...based on fear not science". In addition, the presentation of eye drops has changed fundamentally since the last issue of expiry date guidelines by the DHSS (1975); then most eye drops were dispensed in glass bottles with a separate dropper. Currently the vast majority of eye drop formulations are supplied in plastic bottles with an integral dropper which probably presents a reduced risk of in use contamination compared with the former containers.

Many geriatric patients admitted to hospital are already receiving eye drop therapy for unrelated long term conditions. Current directives dictate that eye drops issued from the hospital pharmacy should be discarded after 7 days of inpatient use which represents a potential waste of resources as the length of stay for such patients is frequently longer than this. Substantial savings could therefore accrue from increasing the in use period to 14 days provided it can be shown that such a move will not subject the patients to an increased risk of infection.

Previous studies into the microbial evaluation of eye drop residues have found no association between the level of contamination and location of use (that is, hospital ward and domicile $)^{5}$ suggesting that the difference in in use expiry periods is unfounded. In addition, it has been demonstrated that the incidence and degree of microbial contamination of eye drops used by hospital inpatients were low and 
Table 1 Incidence of microbial contamination of eye drop residues

\begin{tabular}{llll}
\hline \multirow{2}{*}{ Duration of use } & \multicolumn{3}{l}{ Number of eye drop residues } \\
\cline { 2 - 4 } & Contaminated & Uncontaminated & \% Contaminated \\
\hline 7 days & 21 & 320 & 6.1 \\
14 days & 27 & 268 & 9.1 \\
\hline
\end{tabular}

micro-organisms isolated were predominantly those found on normal skin or as contaminants in the air and were not generally considered to represent a clinically significant threat to the patient. $^{6}$

The aim of this study was to compare the microbial contamination of eye drops residues used by inpatients for both 7 or 14 days in order to assess the validity of an increased in use period for preserved eye drops issued to hospital inpatients.

\section{Materials and methods}

Ethics committee approval was sought and gained before the commencement of the research project. An appropriate number of eye drop residues (minimum 200) were collected from inpatients of Worthing, Southlands, and Brighton General hospitals after 7 days' use. A similar number of residues were accumulated from the same patient base after 14 days' use. Patients who had recently undergone eye surgery were excluded from the study as were those with infective eye disorders. Eye drop residues containing an active drug with inherent antimicrobial properties-for example, Maxitrol (dexamethasone, neomycin and polymyxin), were also rejected from the study. After collection by the research technician, eye drop residues were either analysed immediately or refrigerated at $4^{\circ} \mathrm{C}$ for a maximum of 24 hours before microbial evaluation. The following information was recorded from each eye drop residue retained for analysis:

1 Date

2 Name of patient

3 Approved name and concentration of active drug(s)

4 Name and concentration of preservative(s)

5 Duration (number of days) of use

6 Frequency of use

7 Residual volume and type of bottle in which eye drops were supplied

8 Hospital

Table 2 Micro-organisms identified from eye drop residues after 7 and 14 days' use

\begin{tabular}{|c|c|c|c|}
\hline Duration of use & $\begin{array}{l}\text { Contaminating organism } \\
\text { identified }\end{array}$ & $\begin{array}{l}\text { No of containers } \\
\text { in which they occurred }\end{array}$ & $\%$ Occurrence \\
\hline 7 days & Micrococcus $\mathrm{sp}$ & 6 & 1.8 \\
\hline \multirow[t]{5}{*}{$(\mathrm{n}=341)$} & Staphylococcus epidermidis & 6 & 1.8 \\
\hline & Other staphylococci` & 3 & 0.9 \\
\hline & Bacillus sp & 2 & 0.6 \\
\hline & Penicillium sp & 3 & 0.9 \\
\hline & Cladosporium $\mathrm{sp}$ & 2 & 0.6 \\
\hline 14 days & Micrococcus $\mathrm{sp}$ & 7 & 2.4 \\
\hline \multirow{9}{*}{$(\mathrm{n}=295)$} & Staphylococcus epidermidis & 2 & 0.7 \\
\hline & Other staphylococci* & 3 & 1.0 \\
\hline & Proteus mirabilis & 4 & 1.4 \\
\hline & Serratia liquefaciens & 2 & 0.7 \\
\hline & Bacillus $\mathrm{sp}$ & 5 & 1.7 \\
\hline & Aureobasidium pullulans & 2 & 0.7 \\
\hline & Cladosporium $\mathrm{sp}$ & 3 & 1.0 \\
\hline & Penicillium $\mathrm{sp}$ & 1 & 0.3 \\
\hline & Yeast (unidentified) & 1 & 0.3 \\
\hline
\end{tabular}

$\star$ Includes both coagulase positive and coagulase negative staphylococci.
9 Type of ward (medical or surgical) on which the eye drops were administered.

All analytical work was carried out under aseptic conditions using a laminar airflow cabinet. For each eye drop container, $2 \mathrm{ml}$ of the residual liquid was withdrawn into a sterile syringe and diluted to $10 \mathrm{ml}$ with sterile peptone water containing $1 \%$ Tween 80 and $0.1 \%$ lecithin (as preservative inactivators). The resultant suspension was thoroughly mixed and half of the liquid $(5 \mathrm{ml})$ was then filtered through a $47 \mathrm{~mm}$ mixed cellulose esters membrane $(0.2 \mu \mathrm{m}$ pore size). The membrane was then aseptically transferred to the surface of an overdried tryptone soya agar (TSA) plate and incubated at $30^{\circ} \mathrm{C}$ for up to 7 days. The remaining half of the diluted eye drop residue was similarly filtered, however the membrane was placed on a Sabouraud-dextrose agar (SDA) plate and incubated at $25^{\circ} \mathrm{C}$ for 14 days. TSA was selected as it preferentially supports the growth of bacteria while SDA favours the growth of yeasts and moulds. After incubation the plates were examined, the numbers of colonies counted and the contamination level per $\mathrm{ml}(\mathrm{cfu} / \mathrm{ml})$ of undiluted eye drop residue was calculated. In addition, the contaminants were isolated, subcultured, and identified using conventional microbiological techniques together with API bacterial identification systems. Statistical analyses were performed to determine the significance of differences in the incidence and degree of contamination between the sample groups. Comparisons of the identities of contaminating organisms were also made between the two sample groups.

\section{Results}

In all, 341 inpatient eye drop residues were microbially evaluated following 7 days' use and 295 following 14 days' use. The incidence of microbial contamination of the two groups of eye drop residues is presented in Table 1 .

Although the recorded incidence of microbial contamination was apparently greater for eye drop residues after 14 days' use compared with those after 7 days' use, this difference was not statistically significant ( $p>0.1 \chi^{2}$ test).

The incidence of contamination was $9.4 \%$ (13/139) for eye drops used on surgical wards and $7.0 \%$ (35/497) for those used on medical wards. The difference was not significant ( $p$ $>0.1 \chi^{2}$ test).

The identity of contaminating microorganisms is presented in Table 2. It should be noted that in some cases more than one microorganism was isolated from the eye drop residues. The degree of contamination was estimated by counting the number of colony forming units (cfu) observed on the TSA plates after 7 days' incubation. For those residues which contained viable bacteria, the median level of contaminants (cfu/ml residue) were:

1.0 (range 1-14) after 7 days' use

1.5 (range 1-162) after 14 days' use. The majority ( $>97 \%$ ) of eye drops evaluated in this study contained the quaternary ammonium preservative benzalkonium chloride (BZK) at concentrations varying from $0.004 \%$ to $0.02 \% \mathrm{w} / \mathrm{v}$. The incidence of microbial 
Table 3 Incidence of microbial contamination with respect to concentration of benzalkonium chloride (BZK)

\begin{tabular}{llll}
\hline \multirow{2}{*}{ Conc $(\% w / v)$ BZK } & \multicolumn{2}{l}{ Number of eye drop residues } \\
\cline { 2 - 4 } & Contaminated & Uncontaminated & \% Contaminated \\
\hline$\leqslant 0.005$ & 19 & 122 & 13.5 \\
$\geqslant 0.01$ & 28 & 454 & 5.8 \\
\hline
\end{tabular}

contamination for different concentrations of BZK is shown in Table 3 and was significantly lower for those eye drops containing BZK at concentrations greater than or equal to $0.01 \%$ compared with those at a BZK concentration of less than or equal to $0.005 \%$ ( $p<0.005 \chi^{2}$ test).

\section{Discussion}

The incidences of microbial contamination reported in this project for the 7 and 14 day use eye drop residues; $6.1 \%$ and $9.1 \%$ respectively, were comparable with those from 7 days inpatient use reported by Douch and Davison $(8 \%)^{6}$ and Guest et al $(11.7 \%){ }^{7}$ These values are very much less than the $37 \%$ contamination rate reported by Harte $e t a l,{ }^{8}$ although this latter study was carried out on eye drops presented in glass bottles with separate droppers.

Examination of the types of microbial contamination arising from the two sample groups indicates a broadly similar pattern of micro-organisms. The predominant bacteria isolated were those associated with the normal skin microflora, most notably coagulase negative staphylococci and micrococci. It should be noted, however, that the rate of occurrence of these bacteria was much lower than that which has been reported in the normal, healthy human conjunctiva $(37 \%-94 \%){ }^{9}$ The remainder were micro-organisms frequently found as contaminants in the environment (primarily bacterial and fungal spores). Gram negative bacteria were found in small numbers in samples from the 14 day patient group but were considered to be of doubtful clinical significance.

It was only possible to make an accurate count of the numbers of contaminants for those residues containing bacteria, as the moulds present did not generally grow to produce discrete colony forming units. The level of bacterial contamination (expressed as median concentration of bacterial contaminants $(\mathrm{cfu} / \mathrm{ml})$ ) were similar (1 and 1.5) for the two eye drop groups.

The significantly greater incidence of contamination occurring in eye drop residues with a lower BZK concentration $(0.004 \%-0.005 \%)$ compared with those residues with a higher $\mathrm{BZK}$ concentration $(0.01 \%-0.02 \%)$ is worthy of note. Eye drops with lower concentrations of
BZK also contained the chelating agent disodium edetate. It is claimed that the activity of BZK is amplified in the presence of some chelating agents and it can therefore be used at a concentration as low as $0.004 \%$ in the presence of $0.02 \%$ disodium edetate. ${ }^{10}$ While the incidence of contamination recorded for eye drop residues containing lower concentrations of BZK may be clinically acceptable, this study clearly indicates that the antimicrobial efficacy of BZK at concentrations of $0.01 \%-$ $0.02 \%$ is greater than that for BZK $0.004 \%-$ $0.005 \%$ with disodium edetate.

Samples were collected from both surgical and medical wards in each of the hospitals involved in the study. To ensure that there was no bias in terms of the type of ward the data were analysed and it was found that surgical wards gave rise to a contamination level of $7.0 \%$ overall while medical wards gave rise to $9.4 \%$ overall. These results were subjected to statistical analysis $\left(\chi^{2}\right.$ test) and were found not to be significantly different $(\mathrm{p}>0.1)$.

The results show that (i) there was no statistically significant difference in the incidence of contamination between the 7 and 14 days' use eye drop residues, (ii) the spectrum of micro-organisms isolated was very similar between the groups and was generally skin or air contaminants, and (iii) the levels which were found were low and not significantly different. It can therefore be concluded that there will be no clinically significant increased risk of eye infections if the period of use for eye drops in hospital were to be increased from 7 to 14 days. If the longer duration of use is adopted throughout the UK, an annual saving of approximately $£ 0.5$ million is forecast.

The authors gratefully acknowledge the financial and administrative support of the Research and Development Directorate, South Thames Regional Health Authority and the assistance of the pharmacy staff of Brighton General, Worthing, and Southlands hospitals.

1 Jolly S, ed. British pharmaceutical codex. 8th ed. London: Pharmaceutical Press, 1963 (Suppl) 1966:77.

2 Department of Health and Social Security. Preservation of sterility in ophthalmic preparations in hospitals. Health Service Circular HSC (IS) 122. London: DHSS, 1975.

3 Barkman R, Germanis M, Karpe G, et al. Preservatives in eye drops. Acta Ophthalmol 1969;47:461-75.

4 Wilson J. Eye drops - time for a fresh look at guidelines. Pharm F 1990;3 November: 594.

5 DuBois SK, Pinney RJ, Davison AL. Investigation of the levels of bacterial contamination in used eye drops. Pharm f 1989;30 September:R39.

6 Douch MM, Davison AL. An investigation into the in-use contamination of Timolol eye drops used on the wards. $\mathcal{F}$ Hosp Pharm Practice 1992;2:483-6.

7 Guest H, Hanlon GW, Livingstone DJ. Microbial evaluation of preservative-free eye drops in multiple application containers. Pharm f 1990;245:R17.

8 Harte V, O'Hanrahan M, Timoney R. Microbial contamination in residues of ophthalmic preparations. Int f Pharmaceut 1978;1:165-71.

9 Rosebury T. Microorganisms indigenous to man. New York: McGraw-Hill, 1962:318-23.

10 Lund W, ed. The pharmaceutical codex. 12th ed. London: Pharmaceutical Press, 1994:164-5. 